\title{
Yes-associated protein 1 promotes the metastasis of U251 glioma cells by upregulating Jagged-1 expression and activating the Notch signal pathway
}

\author{
BIN HAO*, XIN CHEN ${ }^{*}$ and YIQUN CAO \\ Department of Brain and Spine Surgery, Fudan University Shanghai Cancer Center, Shanghai 200032, P.R. China
}

Received June 6, 2016; Accepted April 21, 2017

DOI: $10.3892 /$ etm.2018.6322

\begin{abstract}
Yes-associated protein 1 (YAP1) is overexpressed in a number of human cancer types as an oncogene and takes part in processes of tumor cell proliferation, apoptosis and metastasis. The Notch pathway also has an important role in tumorigenesis and progression. However, the possible association between YAP1 and Notch signaling in glioma has remained to be determined. The present study verified the high expression of YAP1, Jagged 1 (JAG1), NOTCH1 and HES1 in the U251 glioma cell line via reverse-transcription quantitative polymerase chain reaction and western blot analysis. The effects of YAP1 knockdown with small interfering RNA and overexpression of JAG1 or HES1 with the respective recombinant plasmid on the expression of these proteins in U251 cells were also assessed. The expression of JAG1, intracellular domain of the notch protein and HES1 was reduced upon knockdown of YAP1, but the expression of YAP1 was not affected by overexpression of JAG1 or HES1. Furthermore, a Transwell assay was used to assess the migration of U251 cells in vitro following knockdown of YAP1 gene and/or overexpression of JAG1 or HES1. U251 cell migration was decreased following YAP1 knockdown, which was compensated by overexpression of JAG1 or HES1. Overexpression of YAP1 upregulated JAG1 to activate Notch signaling in U251 cells and YAP1-dependent activity of JAG1, and the Notch pathway promoted the metastasis of U251 cells in vitro. This suggested that YAP1 promoted the metastasis of U251 cells via upregulating JAG1 and activating Notch signaling.
\end{abstract}

Correspondence to: Dr Bin Hao or Dr Yiqun Cao, Department of Brain and Spine Surgery, Fudan University Shanghai Cancer Center, 270 Dong'an Road, Shanghai 200032, P.R. China

E-mail: hao_beong@163.com

E-mail: caoyq55@163.com

${ }^{*}$ Contributed equally

Key words: Yes-associated protein 1, Jagged 1, notch pathway, overexpression, metastasis, U251 cells

\section{Introduction}

Yes-associated protein 1 (YAP1) is a major downstream effector of the Hippo pathway, which controls organ size, normal tissue homeostasis and stem cell functions by regulating cell proliferation, growth and apoptosis $(1,2)$. YAP1 is negatively regulated by Hippo and upon injury of the body; the activation of YAP1 promotes the proliferation, differentiation and regeneration of damaged tissues $(3,4)$. Overexpression of YAP1 has been found to exist in numerous carcinoma types as an important oncoprotein (5-7); upon its hyperactivation, it greatly promotes cell proliferation and inhibits cell apoptosis. Studies have found that the functional operation of YAP1 involves interactions with other factors. For instance, YAP1 induced the expression of anti-apoptotic genes in cancer cells through forming a complex with T-box 5 and $\beta$-catenin (8). Furthermore, the synergy between YAP1 and KRAS promoted cancer cell invasion and progression (9) and upregulation of YAP1 with abnormal Sonic hedgehog signaling existed in human medulloblastomas (10).

Numerous studies have shown that aberrant Notch signaling was closely associated with the genesis of various cancer types, such as glioma, cervical cancer, melanoma and hepatocellular carcinoma (HCC) (11-14). The complete Notch pathway includes Notch receptors (Notch1, -2, -3 and -4), Notch ligands (Jagged1 and -2 as well as Delta1, -3 and -4 ) and target genes (HES, HEY). Tschaharganeh et al (15) reported that overexpression of YAP1 led to upregulation of Jagged-1 (JAG1), which resulted in activation of the Notch pathway and promoted the proliferation of HCC cells in vitro and in vivo; this YAP-dependent effect in HCC was consistent with that in colorectal and pancreatic cancer.

Orr etal (16) demonstrated that YAP1 was highly expressed in infiltrating astrocytomas and oligodendrogliomas and promoted glioblastoma cell proliferation and metastasis. Notch1 and JAG1 were also demonstrated to be overexpressed in glioma and to have a role in maintaining tumor cell proliferation and survival (17). Therefore, the present study hypothesized that overexpression of YAP1 may promote glioma cell metastasis via upregulation of JAG1 and activation of the Notch pathway. The expression of YAP1, JAG1, NOTCH1 and HES1 in the U251 human glioma cell line and changes in the expression of these proteins after transfection with small interfering (si)RNA targeting YAP1 (siRNA-YAP1) and JAG1 or HES1 overexpression vector were 
therefore assessed. In addition, the cellular migration ability under these conditions was detected. The results indicated that compared with that in normal astrocytes, YAP1 and proteins associated with Notch signaling were upregulated in U251 cells, which was downregulated by siRNA-YAP1 and compensated by overexpression of JAG1 and HES1; these changes were found to be associated with the migratory ability of U251 cells in vitro.

\section{Materials and methods}

Cell lines. The U251 human glioma cell line was obtained from the American Type Culture Collection (Manassas, VA, USA) and cultured in Dulbecco's modified Eagle's medium (HyClone; GE Healthcare, Little Chalfont, UK) supplemented with $10 \%$ fetal calf serum (FCS; Invitrogen; Thermo Fisher Scientific, Inc., Waltham, MA, USA) in a humidified incubator at $37^{\circ} \mathrm{C}$ containing $5 \% \mathrm{CO}_{2}$. Normal astrocytes used in this study were human astrocytes (Sciencell, Carlsbad, CA, USA), which were cultured in complete Astrocyte Medium (Sciencell) in a humidified incubator at $37^{\circ} \mathrm{C}$ containing $5 \% \mathrm{CO}_{2}$.

siRNA synthesis, overexpression vector construction and cell transfection. Two siRNA sequences were designed to target YAP1 (siRNA1, 5'-CCACCAAGCUAGUAAAGAdTdT-3'; siRNA2, 5'-GGUCAGAGAUACUUCUUAAdTdT-3') and a nonsense siRNA with a random sequence (5'-UUCUCC GAACGUGUCACGUTT-3') was used as a negative control. These siRNAs were synthesized by GenePharma (Shanghai, China). JAG1 and HES1 complementary (c)DNA were individually sub-cloned into eukaryotic expression vector, as described previously $(18,19)$. U251 cells were transfected with siRNA and recombinant plasmid using Lipofectamine 2000 (Invitrogen; Thermo Fisher Scientific, Inc.) in accordance with the manufacturer's protocol. U251 cells were collected following $24 \mathrm{~h}$ transfection and co-cultured with the siRNA and/or transfected with recombinant plasmid for $48 \mathrm{~h}$.

Reverse-transcription quantitative polymerase chain reaction $(R T-q P C R)$. In order to determine the relative expression level of YAP1, JAG1, NOTCH1 and HES1 mRNA, total RNA was extracted using TRIzol reagent (Invitrogen; Thermo Fisher Scientific, Inc.) from human astrocytes and U251 cells to perform RT-qPCR. cDNA synthesis was performed using the PrimeScript ${ }^{\mathrm{TM}}$ RT kit (Takara Biotechnology Co., Dalian, China) and qPCR was performed using PrimeScript ${ }^{\mathrm{TM}}$ PCR Master Mix (Takara Biotechnology Co.) in an ABI 7500 real-time PCR system (Applied Biosystems; Thermo Fisher Scientific, Inc.). The PCR cycling conditions were as follows: 40 cycles of $95^{\circ} \mathrm{C}$ for $30 \mathrm{sec}$ and $60^{\circ} \mathrm{C}$ for $30 \mathrm{sec}$. The primers used for PCR are listed in Table I. The $\Delta \Delta \mathrm{Cq}$ method was used for the data analysis (20).

Western blot analysis. The protein levels of YAP1, JAG1, NOTCH1, intracellular domain of Notch protein (NICD) and HES1 in each sample were detected using western blot analysis. Total proteins were extracted with radioimmunoprecipitation assay lysis buffer (Beyotime Institute of Biotechnology, Inc., Shanghai, China) and the protein concentration was determined using a BCA Protein Assay kit (Beyotime Institute of Biotechnology, Inc.). The same
Table I. Sequences of primers used for polymerase chain reaction analysis.

\begin{tabular}{lcl}
\hline Gene & Direction & \multicolumn{1}{c}{ Sequences of primers $\left(5^{\prime}-3^{\prime}\right)$} \\
\hline YAP1 & F & TAGCCCTGCGTAGCCAGTTA \\
& R & GGTTCGAGGGACACTGTAGC \\
JAG1 & F & AAGTGCACCCGCGACG \\
& R & ATTACTGGAATCCCACGCCTC \\
NOTCH1 & F & TGAATGGCGGGAAGTGTGAA \\
& R & ATAGTCTGCCACGCCTCTG \\
HES1 & F & GATAGCTCGCGGCATTCCAA \\
& R & TCGGTATTAACGCCCTCGC \\
& F & ACCTTCTACAATGAGCTGCG \\
& R & CCTGGATAGCAACGTACATGG
\end{tabular}

F, forward; R, reverse; YAP-1, Yes-associated protein 1; JAG1, Jagged 1.

quantity of total protein $(20 \mu \mathrm{g})$ was separated using SDS-PAGE with $7.5 \%$ polyacrylamide prior to transfer onto a nitrocellulose membrane (Biodee Biotechnology, Beijing, China). The membrane was then blocked in Tris-buffered saline containing $0.1 \%$ Tween-20 (TBST) and 5\% nonfat dry milk followed by incubation with primary antibodies overnight at $4^{\circ} \mathrm{C}$. Primary antibodies were anti-YAP1 (cat. no. ab56701), anti-JAG1 (cat. no. ab109536), anti-NOTCH1 (cat. no. ab52627), anti-NICD (cat. no. ab8925), anti-HES1 (cat. no. ab108937) and anti- $\beta$-actin (cat. no. ab1801) (all from Abcam, Cambridge, MA, USA) and were used at the dilutions recommended by the supplier. Subsequent to washing for three times with TBST, membranes were incubated with the secondary antibodies: Horseradish peroxidase-conjugated goat anti-mouse immunoglobulin (Ig)G (dilution, 1:2,000; ab6789) and goat anti-rabbit IgG (dilution, 1:2,000; ab6721) (both from Abcam). Finally, the membranes were washed three times and the protein bands were visualized using an EasyBlot ECL kit (Sangon Biotech Co., Ltd., Shanghai, China) according to the manufacturers protocol and images of the blots were captured using X-ray film.

Cell migration assay. U251 cells with YAP1 knockdown and overexpression of JAG1 or HES1 were used for cell migration assays using the Transwell method. Cells were suspended in serum-free medium $\left(100 \mu \mathrm{l} ; 5 \times 10^{5} / \mathrm{ml}\right)$ and inoculated into the upper chamber with $8-\mu \mathrm{m}$ pore membranes (Corning, NY, USA). Medium containing $10 \%$ FCS was added to the lower chamber, followed by conventional culture for $6 \mathrm{~h}$. The cells were fixed with $4 \%$ formaldehyde and stained with crystal violet after washing with PBS. The medium and cells in the upper chamber were discarded for observing and counting cells on the lower surface of the chamber with a microscope (magnification, x400).

Statistical analysis. Data were analyzed using GraphPad Prism 5.0 software (GraphPad Inc., La Jolla, CA, USA). 
A

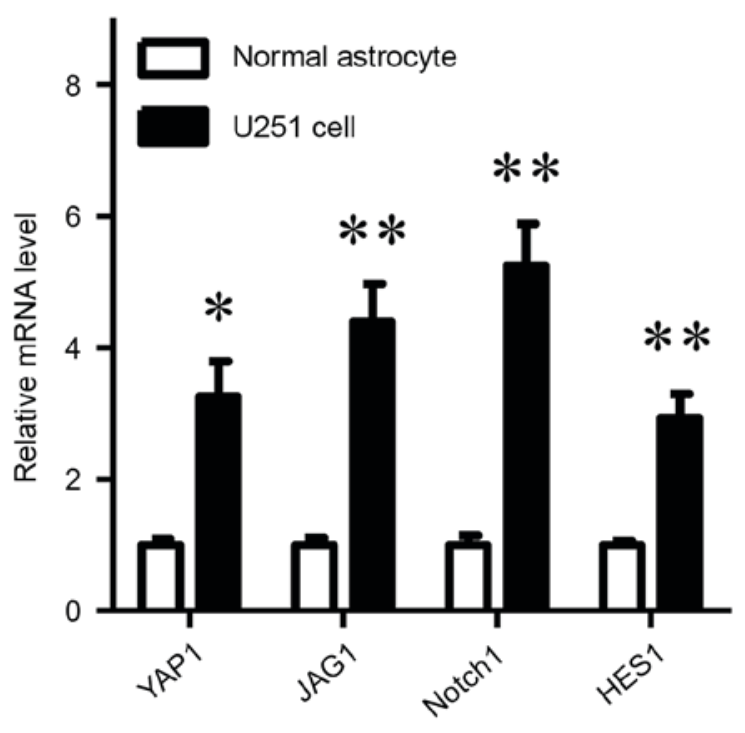

B

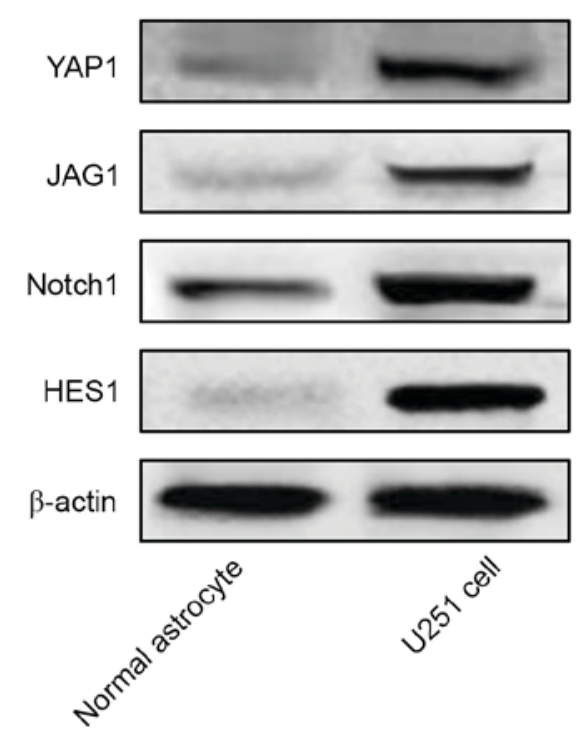

C

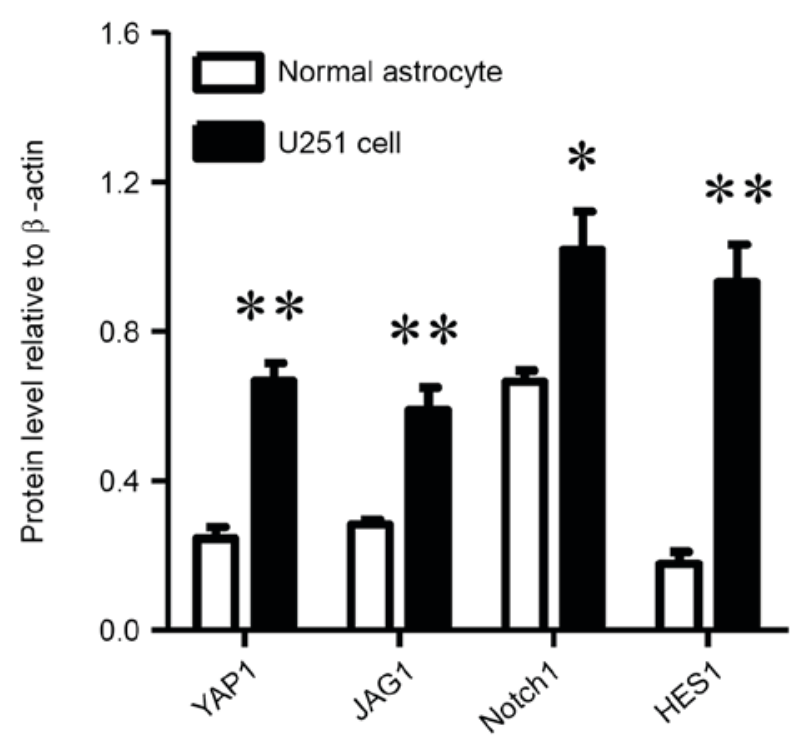

Figure 1. YAP1, JAG1, NOTCH1 and HES1 are upregulated in glioma cells compared with human astrocytes. (A) Relative mRNA levels of YAP1, JAG1, NOTCH1 and HES1 in U251 glioma cells compared with human astrocytes. (B) Western blot analysis of YAP1, JAG1, NOTCH1 and (C) protein levels relative to $\beta$-actin HES1 in U251 glioma cells and human astrocytes. "P $<0.05,{ }^{* *} \mathrm{P}<0.01$, compared with normal astrocytes. YAP-1, Yes-associated protein 1; JAG1, Jagged 1.

Values are expressed as the mean \pm standard deviation from at least three separate experiments. The significance of differences between two groups was detected using Student's t-test. $\mathrm{P}<0.05$ was considered to indicate a statistically significant difference.

\section{Results}

Expression of YAP1, JAG1, NOTCH1 and HES1 in U251 cell line. RT-qPCR and western blot analyses were used to detect the mRNA and protein levels of YAP1, JAG1, NOTCH1 and HES1 in normal astrocytes and U251 glioma cells. As presented in Fig. 1, not only the mRNA levels of YAP1 in U251 cells were obviously higher than those in human astrocytes $(\mathrm{P}<0.05)$, but the mRNA levels of JAG1, NOTCH1 and
HES1 in U251 cells were also significantly higher than those in human astrocytes $(\mathrm{P}<0.01)$. Similarly, the corresponding protein levels were higher in U251 cells, particularly those of YAP1, JAG1 and HES1 $(\mathrm{P}<0.01)$.

Effects of YAP1 knockdown on protein levels in U251 cell line. After $24 \mathrm{~h}$ of transfection with YAP1 siRNA, U251 cells were collected to detect the expression levels of associated proteins using western blot analysis. As presented in Fig. 2A and B, YAP1 siRNA-1 and YAP1 siRNA-2 interfered the YAP1 protein expression, which was obviously lower than that in the blank group and scramble siRNA group $(\mathrm{P}<0.01)$. Furthermore, the NICD and HES1 protein levels in the U251 cells transfected with YAP1 siRNA showed a noticeable decline $(\mathrm{P}<0.01)$; however, the difference in NOTCH1 protein 
A

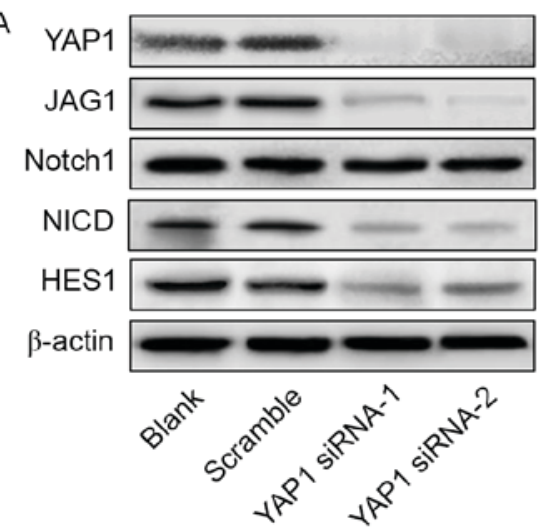

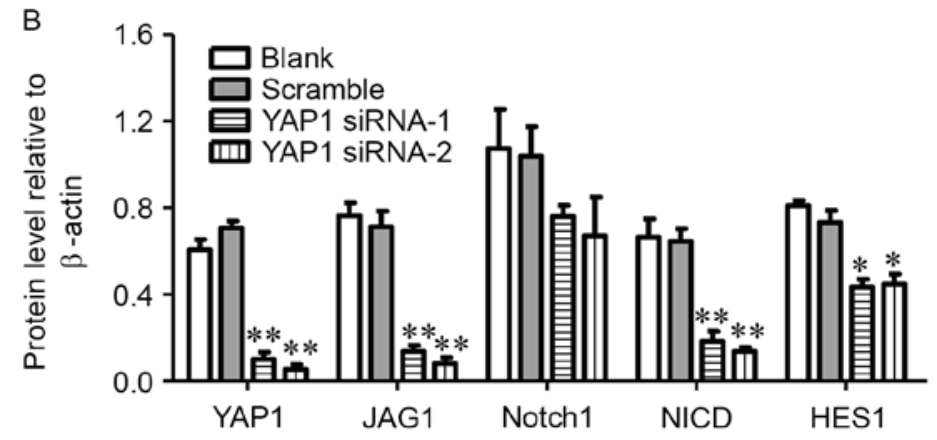

Figure 2. Knockdown of YAP1 leads to downregulation of JAG1, NOTCH1, NICD and HES1 proteins. (A and B) Protein levels of YAP1, JAG1, NOTCH1, NICD and HES1 in blank, scramble siRNA, YAP1 siRNA1 and YAP1 siRNA2 groups. ${ }^{*} \mathrm{P}<0.05,{ }^{* * *} \mathrm{P}<0.01$, compared with scramble group. YAP-1, Yes-associated protein 1; JAG1, Jagged 1; siRNA, small interfering RNA; NICD, intracellular domain of NOTCH protein.

A

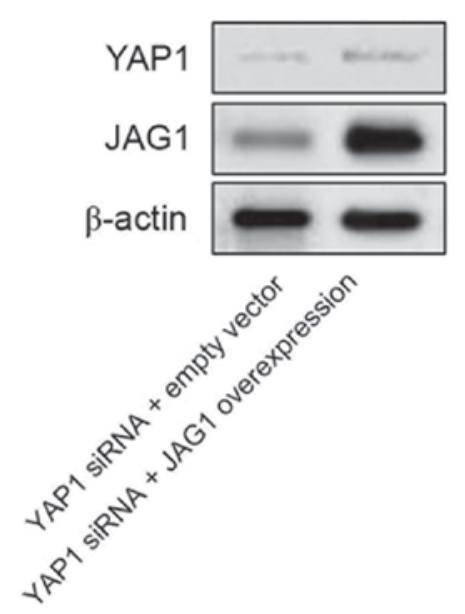

C

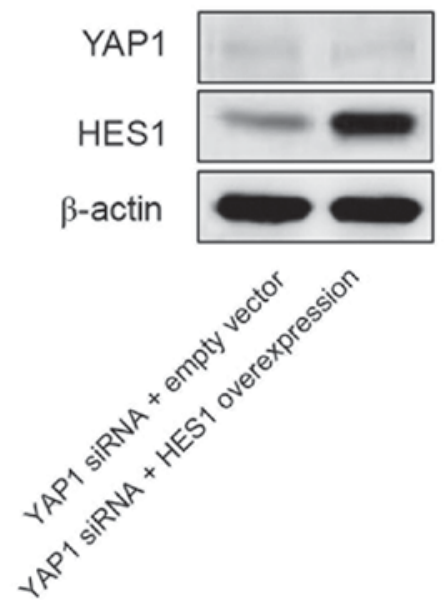

B

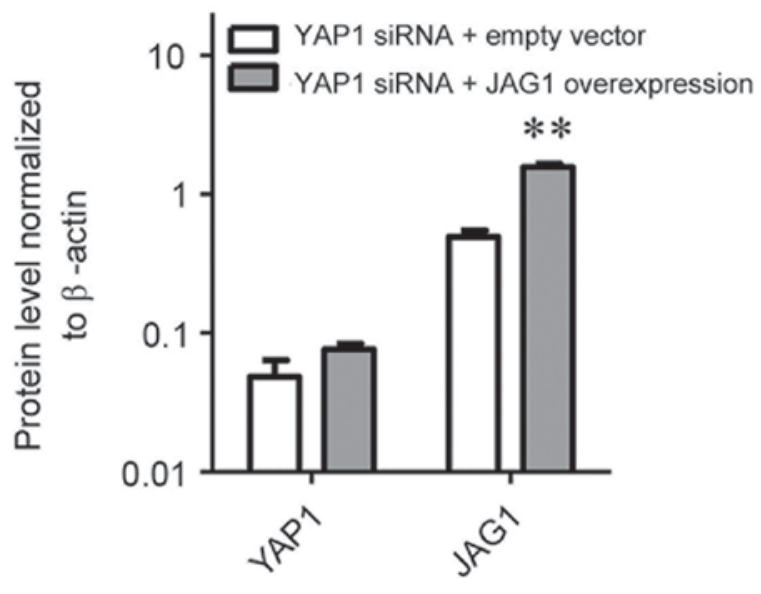

D

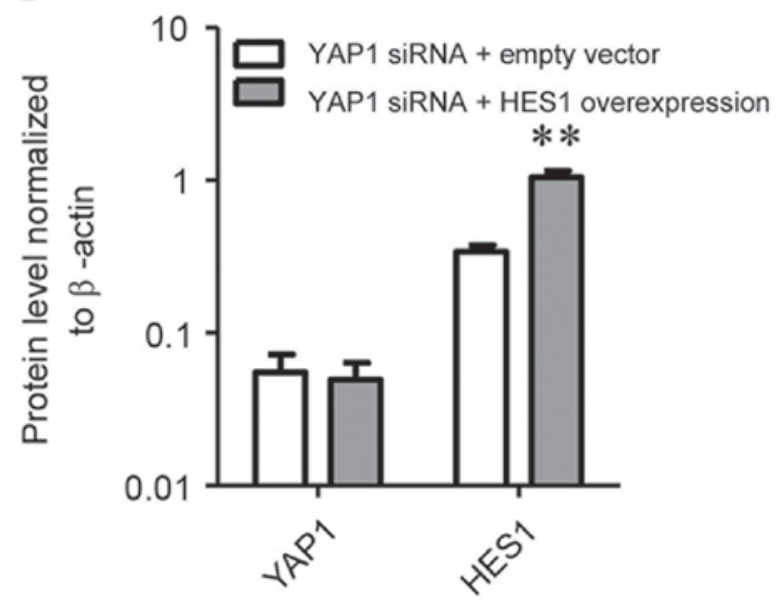

Figure 3. Increased expression of JAG1 or HES1 in U251 cells co-transfected with YAP1 siRNA and overexpression vectors. (A and B) Protein levels of YAP1 and JAG1 in empty vector group and JAG1 overexpression group with knockdown of YAP1 in U251 cells. (C and D) Protein levels of YAP1 and HES1 in empty vector group and HES1 overexpression group with knockdown of YAP1 in U251 cells. ${ }^{* *} \mathrm{P}<0.01$, compared with the group transfected with empty vector. YAP-1, Yes-associated protein 1; JAG1, Jagged 1. 
A

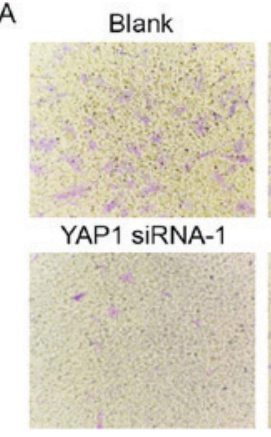

C
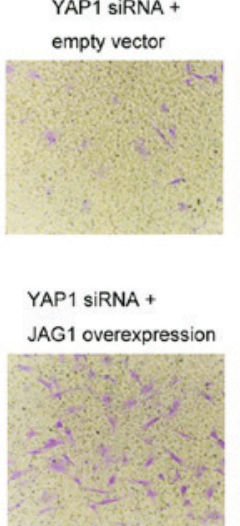

Scramble

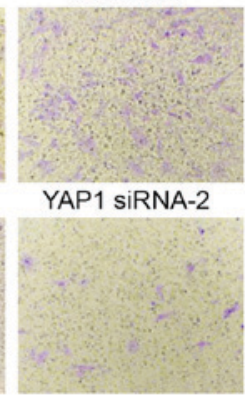

YAP1 SIRNA + empty vector

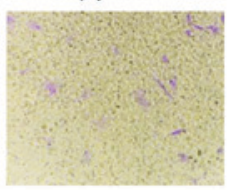

YAP1 SIRNA + HES1 overexpression

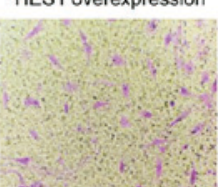

B

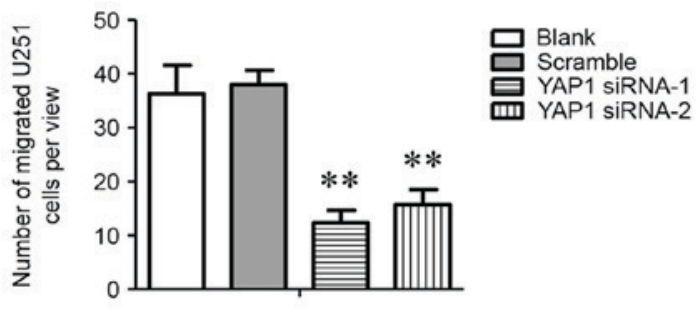

D

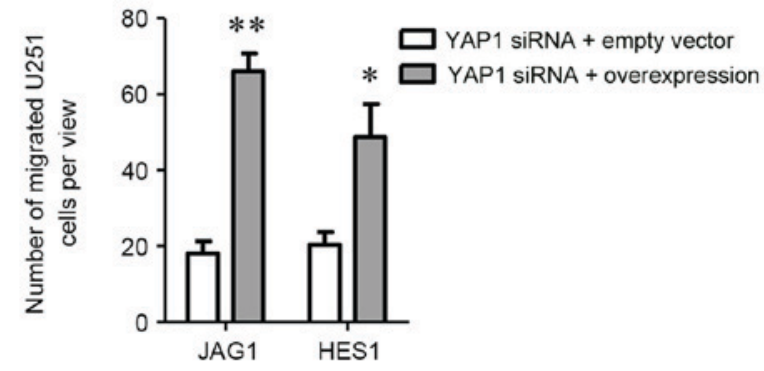

Figure 4. Overexpression of JAG1 or HES1 promotes migration of U251 cells transfected with YAP1 siRNA. (A) U251 cell migration in blank, scramble siRNA, YAP1 siRNA1 and YAP1 siRNA2 groups (magnification, 200x); (B) Number of migrated U251 cells per field of view in blank, scramble siRNA, YAP1 siRNA1 and YAP1 siRNA2 groups. (C) U251 cell migration in empty vector group, JAG1 overexpression group and HES1 overexpression group with knockdown of YAP1 in U251 cells (magnification, x200); (D) number of migrated U251 cells per field of view in empty vector group, JAG1 overexpression group and HES1 overexpression group with knockdown of YAP1 in U251 cells. ${ }^{*} \mathrm{P}<0.05,{ }^{* *} \mathrm{P}<0.01$, compared with the group transfected with empty vector or blank group. YAP-1, Yes-associated protein 1; JAG1, Jagged 1.

levels between YAP1 siRNA group, blank group and scramble siRNA group was not significant.

Effects of overexpression of JAG1 and HES1 on U251 cells following YAPl gene knockdown. Recombinant plasmids for overexpressing JAG1 or HES1 were respectively transfected into U251 cells following YAP1 gene knockdown and the expression of YAP1, JAG1 and HES1 protein were investigated. When U251 cells were co-transfected with recombinant plasmids and siYAP1, overexpression of JAG1 or HES1 replenished the decrease of JAG1 and HES1 in U251 cells following YAP1 gene knockdown $(\mathrm{P}<0.01)$. However, neither overexpression of JAG1 or HES1 reversed the downregulation of YAP1 (Fig. 3A-D).

Migration of the U251 cell line following YAP1 gene knockdown and overexpression of JAG1 and HES1. The migration ability of U251 cells was assessed via the Transwell method. Following knockdown of YAP1, the number of migrated cells per field of view was significantly reduced compared with that in the blank group and scrambled siRNA group (12 or 15 vs. 36 and 38, respectively; $\mathrm{P}<0.01$; Fig. $4 \mathrm{~A}$ and $\mathrm{B}$ ). This reduction caused by knockdown of YAP1 was compensated by overexpression of JAG1 or HES1 when cells were co-transfected with siYAP1 and recombinant plasmids and there were significant increases between empty vector and JAG1 overexpression (18 vs. 64 ; $\mathrm{P}<0.01$ ) or empty vector and HES1 overexpression groups (20 vs. 46; $\mathrm{P}<0.05$; Fig. $4 \mathrm{C}$ and $\mathrm{D}$ ).

\section{Discussion}

YAP1 exerts its growth stimulatory effects via forming a complex with Tafazzin, which promotes proliferation and inhibits apoptosis. Phosphorylation of YAP1 suppresses proliferation and accelerates apoptosis. Overexpression of YAP1 accounts for the overproliferation in numerous solid tumor types and has an important role in tumorigenesis and tumor progression $(21,22)$. The present study demonstrated that YAP1 is highly expressed in U251 glioma cells compared with that in normal astrocyte cells, which conformed to the overexpression of YAP1 in infiltrating astrocytomas and oligodendrogliomas (16). Furthermore, compared with that in normal astrocytes, the U251 cell line was found to exhibit high expression of JAG1, NOTCH1 and HES1 mRNA and protein, which are ligand, receptor and downstream response gene of Notch signaling, respectively $(23,24)$. The Notch signaling pathway is deregulated in numerous types of solid tumor and it directly or indirectly influences cancer. NOTCH1 has been previously reported to be expressed at a high level in human glioma and to rise with the pathological grade (25).

A previous study showed that YAP1 upregulated JAG1 and activated the Notch signaling in HCC cells (15). In order to elucidate the association between YAP1, JAG1 and Notch signaling in U251 cells, the present study used a knockdown approach with siRNA to directly target YAP1 mRNA in the U251 cell line, which led to a decrease of YAP1, JAG1, NICD and HES1. 
NICD is the activated form of NOTCH1 after binding to its ligand and has a role in the transmission of signals via entry into the nucleus (26). This indicated that YAP1 positively regulated JAG1 and Notch signaling in U251 cells in vitro.

The Notch pathway has been proved to take part in the invasion and metastasis of tumors; for instance, high expression of JAG1 and NOTCH1 promoted the invasion and metastasis of breast cancer cells (27) and overexpression of NOTCH1 and HES1 was also responsible for the invasion and metastasis of HCC cells (28). The present study demonstrated that knockdown of YAP1 decreased U251 cell migration and that overexpression of JAG1 or HES1 enhanced cell migration of U251 cells transfected with YAP1 siRNA, which indicated that Notch signaling is associated with U251 cell migration in vitro. At the same time, YAP1 knockdown led to downregulation of JAG1 and inactivated Notch signaling, while overexpression of JAG1 or HES1 did not influence the expression of YAP1.

In conclusion, the present study demonstrated that YAP1, JAG1, NOTCH1 and HES1 were highly expressed in U251 cells compared to normal astrocytes and that the expression of JAG1, NICD and HES1 was significantly reduced in U251 cells with knockdown of YAP1, which also reduced the cell migratory capacity in vitro. Overexpression of JAG1 or HES1 enhanced cell migration in vitro, but did not impact the expression of YAP1. Taken together, these findings indicated that upregulation of JAG1 activated Notch signaling and promoted U251 cell migration in vitro. Furthermore, YAP1 was overexpressed in U251 cells and associated with high JAG1 expression to take part in the activation of Notch signaling and cell migration in vitro. These findings contributed to the understanding of the mechanisms of glioma cell metastasis and may provide approaches for the treatment of gliomas.

\section{Acknowledgments}

This study was financially supported by the Shanghai Natural Science Foudation (grant no. 16ZR1406300) and the Natural Science Foundation of China (grant no. 81572921).

\section{Competing interests}

The authors declare that they have no competing interests.

\section{References}

1. Camargo FD, Gokhale S, Johnnidis JB, Fu D, Bell GW, Jaenisch R and Brummelkamp TR: YAP1 increases organ size and expands undifferentiated progenitor cells. Curr Biol 17: 2054-2060, 2007.

2. Cao X, Pfaff SL and Gage FH: YAP regulates neural progenitor cell number via the TEA domain transcription factor. Genes Dev 22: $3320-3334,2008$

3. Johnson R and Halder G: The two faces of Hippo: Targeting the Hippo pathway for regenerative medicine and cancer treatment. Nat Rev Drug Discov 13: 63-79, 2014.

4. Cai J, Zhang N, Zheng Y, de Wilde RF, Maitra A and Pan D: The Hippo signaling pathway restricts the oncogenic potential of an intestinal regeneration program. Genes Dev 24: 2383-2388, 2010.

5. Liu H, Jiang D, Chi F and Zhao B: The Hippo pathway regulates stem cell proliferation, self-renewal, and differentiation. Protein Cell 3: 291-304, 2012.

6. Harvey KF, Zhang X and Thomas DM: The Hippo pathway and human cancer. Nat Rev Cander 13: 246-257, 2013.

7. Zhao B, Tumaneng K and Guan KL: The Hippo pathway in organ size control, tissue regeneration and stem cell self-renewal. Nat Cell Biol 13: 877-883, 2011.
8. Rosenbluh J, Nijhawan D, Cox AG, Li X, Neal JT, Schafer EJ, Zack TI, Wang X, Tsherniak A, Schinzel AC, et al: $\beta$-catenin-driven cancers require a YAP1 transcriptional complex for survival and tumorigenesis. Cell 151: 1457-1473, 2012.

9. Shao DD, Xue W, Krall EB, Bhutkar A, Piccioni F, Wang X, Schinzel AC, Sood S, Rosenbluh J, Kim JW, et al: KRAS and YAP1 converge to regulate EMT and tumor survival. Cell 158: 171-184, 2014.

10. Fernandez LA, Northcott PA, Dalton J, Fraga C, Ellison D, Angers S, Taylor MD and Kenney AM: YAP1 is amplified and up-regulated in hedgehog-associated medulloblastomas and mediates Sonic hedgehog-driven neural precursor proliferation. Genes Dev 23: 2729-2741, 2009.

11. Katoh M: Networking of WNT, FGF, Notch, BMP, and Hedgehog signaling pathways during carcinogenesis. Stem Cell Rev 3: 30-38, 2007.

12. Yao J, Duan L, Fan M, Yuan J and Wu X: Notch1 induces cell cycle arrest and apoptosis in human cervical cancer cells: Involvement of nuclear factor kappa B inhibition. Int J Gynecol Cancer 17: 502-510, 2007.

13. Liu ZJ, Xiao M, Balint K, Smalley KS, Brafford P, Qiu R, Pinnix CC, $\mathrm{Li}$ X and Herlyn M: Notch1 signaling promotes primary melanoma progression by activating mitogen-activated protein kinase/phosphatidylinositol 3-kinase-Akt pathways and up-regulating $\mathrm{N}$-cadherin expression. Cancer Res 66: 4182-4190, 2006.

14. Fan RH, Li J, Wu N and Chen PS: Late SV40 factor: A key mediator of Notch signaling in human hepatocarcinogenesis. World $\mathrm{J}$ Gastroenterol 17: 3420-3430, 2011.

15. Tschaharganeh DF, Chen X, Latzko P, Malz M, Gaida MM, Felix K, Ladu S, Singer S, Pinna F, Gretz N, et al: Yes-associated protein up-regulates Jagged-1 and activates the Notch pathway in human hepatocellular carcinoma. Gastroenterology 144: 1530-1542e12, 2013.

16. Orr BA, Bai H, Odia Y, Jain D, Anders RA and Eberhart CG: Yes-associated protein 1 is widely expressed in human brain tumors and promotes glioblastoma growth. J Neuropathol Exp Neurol 70: 568-577, 2011.

17. Purow BW, Haque RM, Noel MW, Su Q, Burdick MJ, Lee J, Sundaresan T, Pastorino S, Park JK, Mikolaenko I, et al: Expression of Notch-1 and its ligands, Delta-like-1 and Jagged-1, is critical for glioma cell survival and proliferation. Cancer Res 65: 2353-2363, 2005.

18. Li BY, Ma CG, Zhao HY, Zhao CL, Xu QY and Gao FL: Construction of eukaryotic expression vector containing rat Hes1 gene and expression in neural precursor cells for the study of its differentiation functions. Acta Anatomica Sinica 40: 187-192, 2009.

19. Qian DH, Wu XJ, Jiang H, Kuang CY, Wang K, Song MB and Huang L: Construction of eukaryotic vector expressing Jagged1 gene and its effect on proliferation and apoptosis of vascular smooth muscle cells. J Third Mil Med Univ 33: 1095-1098, 2011.

20. Livak KJ and Schmittgen TD: Analysis of relative gene expression data using real-time quantitative PCR and the 2(-Delta Delta C(T)) method. Methods 25: 402-408, 2001.

21. Steinhardt AA, Gayyed MF, Klein AP, Dong J, Maitra A, Pan D, Montgomery EA and Anders RA: Expression of Yes-associated protein in common solid tumors. Hum Pathol 39: 1582-1589, 2008.

22. Xu MZ, Yao TJ, Lee NP, Ng IO, Chan YT, Zender L, Lowe SW, Poon RT and Luk JM: Yes-associated protein is an independent prognostic marker in hepatocellular carcinoma. Cancer 115: 4576-4585, 2009.

23. Dohda T, Maljukova A, Liu L, Heyman M, Grandér D, Brodin D, Sangfelt O and Lendahl U: Notch signaling induces SKP2 expression and promotes reduction of p27Kip1 in T-cell acute lymphoblastic leukemia cell lines. Exp Cell Res 313: 3141-3152, 2007.

24. Bridges $E$, Oon $C E$ and Harris A: Notch regulation of tumor angiogenesis. Future Oncol 7: 569-588, 2011.

25. Yi HB, Shi SS, Yang WZ and Chen CM: The expression and significance of notch-1 gene in human gliomas. Cancer Res Prev Treat 33: 701-703, 2006 (In Chinese).

26. Wang LX and Hua ZC: Research progress of the Notch signaling pathway. Chin Med Biotechnol 4: 224-226, 2009 (In Chinese).

27. Reedijk M, Odorcic S, Chang L, Zhang H, Miller N, McCready DR, Lockwood G and Egan SE: High-level coexpression of JAG1 and NOTCH1 is observed in human breast cancer and is associated with poor overall survival. Cancer Res 65: 8530-8537, 2005.

28. Cantarini MC, de la Monte SM, Pang M, Tong M, D'Errico A, Trevisani F and Wands JR: Aspartyl-asparagyl beta hydroxylase over-expression in human hepatoma is linked to activation of insulin-like growth factor and notch signaling mechanisms. Hepatology 44: 446-457, 2006. 